Lovelock gravity and classical wormholes

This content has been downloaded from IOPscience. Please scroll down to see the full text. 1991 Class. Quantum Grav. 8935

(http://iopscience.iop.org/0264-9381/8/5/017)

View the table of contents for this issue, or go to the journal homepage for more

Download details:

IP Address: 161.111.22.141

This content was downloaded on 11/12/2013 at $11: 31$

Please note that terms and conditions apply. 


\title{
Lovelock gravity and classical wormholes
}

\author{
G A Mena Marugán \\ Instituto de Optica 'Daza de Valdés', Consejo Superior de Investigaciones Científicas \\ (CSIC), Serrano 121, 28006 Madrid, Spain
}

Received 2 August 1990, in final form 4 December 1990

\begin{abstract}
We first consider Lovelock gravity as a perturbative theory and then apply the results to a $D$-dimensional homogeneous and isotropic minisuperspace model provided with matter fields for which there exist instanton solutions. We develop a general procedure to analyse these solutions, which may be interpreted as representing a tunnelling. Adding some reasonable restrictions to the Lovelock coefficients, it is shown that the Lovelock corrections preserve the essential feature of Einstein gravity models of giving rise to an essentially unique instanton solution.
\end{abstract}

\section{Introduction}

The study of spacetimes of more than four dimensions leads to the consideration of Lovelock gravity as a natural generalization of Hilbert-Einstein general relativity.

The Lovelock Lagrangian is formed by a linear combination of dimensionally extended Euler densities [1], which can be considered as the topological generalization of the Hilbert-Einstein Lagrangian. Moreover, the classical dynamical equations associated with the Lovelock Lagrangian are of the general relativity type [2]. On the other hand, the low energy limit of supergravity theories derived from strings predicts higher order curvature corrective terms added to the Hilbert-Einstein Lagrangian [3]. Zwiebach [3] and Zumino [4] showed that if these higher order curvature terms give rise to the dimensionally extended Euler densities of order larger than unity, the corresponding low energy limit of the superstring theory turns out to be ghost-free.

Quadratic and higher order curvature terms are corrections to the Einstein Lagrangian in the low energy limit, in which Einstein gravity provides a valid approximation. It has been recently proposed that Lovelock theories should be treated in a perturbative way $[5,6]$. This could open a way to solve the problems that these kinds of theories present, for example:

(i) The existence of several families of classical solutions allows the system to jump from one family to another in a random way [7]; among these families of solutions, only one should correspond to the perturbed Einstein solutions.

(ii) The multi-valuedness in the inversion of the relation between the metric time derivatives and their associated momenta [7, 8]; if we interpret Lovelock gravity from a perturbative point of view, then this inversion should be uniquely defined.

(iii) The existence of initial data sets for which the Cauchy problem is ill posed, leading to an unpredictable evolution [9]. Perturbatively, however, the classical evolution should always be well defined. 
In this paper, we shall study a $D$-dimensional $(D \geq 3)$ homogeneous and isotropic minisuperspace model provided with matter fields for which there exist instanton type solutions. We first consider Lovelock gravity as a perturbative theory for our minisuperspace model in section 2 . In section 3 we include the matter content of the system and analyse the corresponding classical Euclidean solutions.

\section{Lovelock gravity as a perturbative theory}

The Lovelock gravitational action for a $D$-dimensional manifold $\mathcal{M}$ can be expressed $[1,2]$ :

$S=\int_{\mathcal{M}} \sum_{m=1}^{M} \frac{L_{m}}{(D-1) !(D-2 m)} \epsilon_{a_{2} \ldots a_{D}} R^{a_{3} a_{2}} \ldots R^{a_{2 m-1} a_{2 m}} e^{a_{2 m+1}} \ldots e^{a_{D}}$.

Here, $\left\{e^{a}\right\}, a=1, \ldots, D$, is the $D$-tetrad associated with the manifold $\mathcal{M}$ and $R_{b}^{a}$ is the corresponding curvature 2-form [10], and $\epsilon_{a_{1} \ldots a_{D}}$ is the Levi-Civita tensor; the $L_{m}$ are generic real constants and $M$ is an integer number related to the dimension of the manifold by

$$
M= \begin{cases}(D-2) / 2 & \text { for } D \text { even } \\ (D-1) / 2 & \text { for } D \text { odd }\end{cases}
$$
take

As the first term in (1) must correspond to the Hilbert-Einstein Lagrangian, we

$$
L_{1}=\frac{(D-1)(D-2)}{16 \pi G_{D}}
$$

with $G_{D}$ the $D$-dimensional gravitational constant. $G_{D}$ must not be confused with the effective four-dimensional gravitational constant for universes in which $(D-4)$ dimensions are compactified. In any case, assuming all Lovelock terms $(m>1)$ to be corrections to the Hilbert-Einstein action in the low energy limit, one must require $L_{1}$ to be positive; otherwise, the gravity theory would not be attractive in that limit.

In this work we will restrict ourselves to the minisuperspace:

$$
\mathrm{d} s^{2}=-N^{2}(t) \mathrm{d} t^{2}+a^{2}(t) \mathrm{d} \Omega_{D-1}^{2}
$$

where $N(t)$ stands for the lapse function, $a(t)$ is the scale factor and $\mathrm{d} \Omega_{D-1}^{2}$ denotes a line element on the unit $(D-1)$-sphere.

$D$-dimensional isotropic spacetimes have been previously considered in searching for instanton solutions of Einstein gravity coupled to different types of matter fields $[11,12]$, and in the study of other Lovelock models [13-17]. D-dimensional anisotropic minisuperspaces, which could eventually lead to compactification of the extra $(D-4)$ dimensions, will be considered elsewhere.

For our minisuperspace, we get the Lovelock action:

$$
\begin{array}{rl}
S=A_{D-1} \int \mathrm{d} & t N a^{D-1} \sum_{m=1}^{M} L_{\bar{m}}\left[\frac{2 m}{\bar{D}-2 m} \frac{1}{a N} \frac{\mathrm{d}}{\mathrm{d} t}\left(\frac{\dot{a}}{N}\right)\left(\frac{1}{a^{\overline{2}}}+\frac{\dot{a}^{2}}{a^{\overline{2}} N^{2}}\right)^{m-1}\right. \\
& \left.+\left(\frac{1}{a^{2}}+\frac{\dot{a}^{2}}{a^{2} N^{2}}\right)^{m}\right]
\end{array}
$$


where $A_{D-1}=2 \pi^{D / 2} / \Gamma(D / 2)$ is the area of the unit $(D-1)$-sphere.

Taking into account surface terms, the Lovelock action (5) becomes $[6,18]$ :

$S=L_{1} A_{D-1} \int \mathrm{d} t N a^{D-1}\left[P\left(\frac{1+\dot{a}^{2} / N^{2}}{a^{2}}\right)-\frac{2 \dot{a}^{2}}{a^{2} N^{2}} \int_{0}^{1} \mathrm{~d} x Q\left(\frac{1+x^{2} \dot{a}^{2} / N^{2}}{a^{2}}\right)\right]$

where, following Whitt [17] and denoting $\hat{L}_{m}=L_{m} / L_{1}$, we have associated a polynomial $P(x)$ to our gravitational theory

$$
P(x)=\sum_{m=1}^{M} \hat{L}_{m} x^{m}
$$

with derivative

$$
Q(x)=\sum_{m=1}^{M} \hat{L}_{m} m x^{m-1} .
$$

In the case of asymptotically flat spaces, in order to render the total surface contribution finite $[19,20]$, one should include additional surface terms which have the same expression as the previous ones, but evaluated for the boundary imbedded in flat space. We can ignore them for the moment, as they do not affect the dynamical equations.

From (6), the gravitational momentum associated to the scale factor can be expressed by [6]

$$
p=-2 L_{1} A_{D-1} a^{D-3} \frac{\dot{a}}{N} \int_{0}^{1} \mathrm{~d} x Q\left(\frac{1+x^{2} \dot{a}^{2} / N^{2}}{a^{2}}\right) \equiv V\left(\frac{\dot{a}}{N}, a\right) .
$$

The matter content of the theory will be described by a generic action, $S_{\text {mat }}=$ $\int \mathrm{d} t \mathcal{L}_{\text {mat }}$, where we can include the cosmological term.

Taking into account that for homogeneous and isotropic models,

$$
\frac{1}{a^{D-1} A_{D-1}} \frac{\partial \mathcal{L}_{\mathrm{mat}}}{\partial N}=-\frac{T_{11}}{N^{2}}
$$

$T_{11}$ being the timelike component of the energy-momentum tensor for the matter content of the system, the Hamiltonian constraint can then be written as [6]

$$
P\left(\frac{1+\dot{a}^{2} / N^{2}}{a^{2}}\right)=\frac{T_{11}}{L_{1} N^{2}}
$$

In general, we will first solve the dynamical equations for the matter fields of the system, evaluating $T_{11}$ on the corresponding solutions and studying then the gravitational dynamics of the model.

There exist several families of classical solutions to the gravitational dynamics (11), for $P(x)$ is a polynomial of degree $M$ which is larger than unity. Therefore (11) can be interpreted as a functional relation between $\left(1+\dot{a}^{2} / N^{2}\right) / a^{2}$ and $T_{11} / L_{1} N^{2}$, which defines an algebraic function of $M$ branches [21],

$$
1+\frac{\dot{a}^{2}}{N^{2}}=a^{2} P_{i}^{-1}\left(\frac{T_{11}}{L_{1} N^{2}}\right) \quad i=1, \ldots, M .
$$


Thus, (12) leads to $M$ different dynamical equations.

On the other hand, the relation between the time derivative of the scale factor and the corresponding momentum is not monovalued. Equation (9) can be thought of as a functional relation between $\dot{\alpha} / N$ and $p$ for fixed $a$. Since $V$ is a polynomial of degree $2 M-1$ in $\dot{a} / N$, this relation defines $\dot{a} / N$ as an algebraic function of $2 M-1$ branches of $p, \dot{a} / N=V_{i}^{-1}(p, a), i=1, \ldots, 2 M-1$, so that the global inversion is not possible.

The Lagrange equation for the scale factor can be written

$$
0=\frac{\mathrm{d}}{\mathrm{d} t}\left(\frac{\partial \mathcal{L}}{\partial \dot{a}}\right)-\frac{\partial \mathcal{L}}{\partial a}=\frac{\partial p}{\partial \dot{a}} \ddot{a}+\frac{\partial p}{\partial a} \dot{a}-\frac{\partial \mathcal{L}}{\partial a} .
$$

Given a certain initial data set verifying the Hamiltonian constraint (11) on a constant time surface, equation (13) will fix the scale factor evolution provided that $\partial p / \partial \dot{a} \neq 0$ for the initial values of $a$ and $\dot{a}$. Thus, the Cauchy problem is ill posed only for initial data which does not even allow the local inversion of the derivative-momentum relation (9).

As previously mentioned, all these problems can be solved in a purely perturbative treatment of the theory. This would mean that the only physically relevant classical solution is the perturbed Einstein solution; in the vicinity of this solution, the inversion of the relation (9) is possible and, consequently, the Cauchy problem turns out to be always well posed for initial data corresponding to this solution.

We can select this perturbative classical solution if we realize that, when no matter is present, the flat Euclidean Einstein solution remains unmodified in the presence of the Lovelock perturbations. Thus, we choose in (12) the branch $P_{1}^{-1}$ defined by the condition

$$
P_{1}^{-1}(z=0)=0 .
$$

Condition (14) uniquely fixes the inversion branch $P_{1}^{-1}[6]$. For this inversion to be well defined for any positive value of the Lorentzian energy density $T_{11} / L_{1} N^{2}$, we must require of the polynomial $P(x)$ to be a strictly increasing function in the whole real semi-axis $x \geq 0$ [6]. From now on, we will assume that this requirement is fulfilled by our polynomial $P(x)$.

A similar requirement was also considered by Whitt [17], who showed that, for Lovelock gravity without a cosmological constant, there exists a perturbed Schwarzschild-type solution only if the polynomial $P(x)$ is a strictly increasing function in all $x \geq 0$ and that this solution is the only spherically symmetric black hole solution, preventing the appearance of naked singularities, with positive black hole mass and gravitational constant.

Denoting $x_{1}=\max \{x \in \mathbb{R} / Q(x)=0\}<0$, it is easy to see that $P_{1}^{-1}(z)$ is well defined by analytic continuation from $z=0$ along the real axis for all $z>z_{1}=P\left(x_{1}\right)$ $\left(z_{1}<0\right)$. For $z \in\left(z_{1}, \infty\right)$, we have $P_{1}^{-1}(z) \in\left(x_{1}, \infty\right)$ and, thus, $\dot{a}^{2} / N^{2} \in\left(a^{2} x_{1}-1, \infty\right)$ (with $a^{2}>0$ ) [6].

We can also select the perturbative inversion of the derivative-momentum relation (9) by taking into account that the correspondence between $\dot{a} / N=0$ and $p=0$ remains unmodified when the Lovelock perturbations are introduced. So, we choose the inversion branch of (9) which verifies

$$
V_{1}^{-1}(p=0, a)=0 .
$$


As

$$
\partial_{\dot{a} / N} V\left(\frac{\dot{a}}{N}, a\right)=-2 L_{1} A_{D-1} a^{D-3} Q\left(\frac{1+\dot{a}^{2} / N^{2}}{a^{2}}\right)
$$

the inversion defined by (15) is possible for all fixed real $a \neq 0$, because then, $\partial_{\dot{a} / N} V(\dot{a} / N=0, a) \neq 0$. Using similar arguments to those in [6] it is easily shown that the so defined inversion can be analytically continued from $p=0$ to the entire real axis, $p \in \mathbb{R}$, in such a way that $V_{1}^{-1}(\mathbb{R}, a)$ is equal to $\mathbb{R}$. This inversion can also be continued analytically along the imaginary axis from $p=0$ up to $p= \pm \mathrm{i} p_{1}$, where $\pm \mathrm{i} p_{1}=V\left( \pm \mathrm{i} \sqrt{1-a^{2} x_{1}}, a\right)$, and it is readily seen that, for $p \in\left(-\mathrm{i} p_{1},+\mathrm{i} p_{1}\right)$, $V_{1}^{-1}(p, a) \in\left(-\mathrm{i} \sqrt{1-a^{2} x_{1}},+\mathrm{i} \sqrt{1-a^{2} x_{1}}\right)$.

Thus, the range of $\dot{a}^{2} / N^{2}=\left(V_{1}^{-1}(p, a)\right)^{2}$ for $p \in \mathbb{R} \cup\left(-i p_{1},+i p_{1}\right)$ and fixed nonvanishing real $a$ equals the interval $\left(a^{2} x_{1}-1, \infty\right)$, which is the interval of definition of the scale factor derivative on the perturbative classical solutions if $T_{11} / L_{1} N^{2}>$ $z_{1}$; then, our Lovelock model is perturbatively well defined in the region $a^{2}>0$, $T_{11} / L_{1} N^{2}>z_{1}$.

Since $V_{1}^{-1}(p, a)=-V_{1}^{-1}(-p, a)$ for $p \in \mathbb{R} \cup\left(-\mathrm{i} p_{1},+\mathrm{i} p_{1}\right)$, it can also be seen that $\dot{a}^{2} / N^{2}=\left(V_{1}^{-1}(p, a)\right)^{2}$ is, in fact, a function of $p^{2}$ properly defined for all $p^{2} \in\left(-p_{1}^{2}, \infty\right)$.

In order to clarify the correspondence between $P_{1}^{-1}(z), V_{1}^{-1}(p, a)$ and the perturbative inversions, it is convenient to introduce explicitly a perturbative parameter $\beta$ in the Lovelock corrections. Thus, we rewrite the Lovelock coefficients: $\hat{L}_{m}=l_{m} \beta^{m-1}$, so that the $m$ th term in the Lovelock Lagrangian is a perturbative correction of the preceding $m-1$ terms. The perturbative solutions can be characterized by admitting a Taylor series in the perturbative parameter $\beta[5,22]$. The perturbative inversion of $(9)$ is then obtained by inserting the substitution

$$
\left(\frac{\dot{a}}{N}\right)_{\text {pert }}=\sum_{m=0}^{\infty} \beta^{m} \dot{a}_{m}
$$

After some tedious calculations, we get $\dot{a}_{m} \propto p$ for all $m$; so $\left[(\dot{a} / N)_{\text {pert }}\right]_{\mid p=0}=0$, which is precisely condition (15) on $V_{1}^{-1}$. For fixed $a$ and $\beta$, the series (17) would converge in some neighbourhood of $p=0$; beyond the convergence radius, the inversion given by (17) could be analytically continued. Since $(\dot{a} / N)_{\text {pert }}$ and $V_{1}^{-1}$ coincide around $p=0, V_{1}^{-1}$ is precisely the result of such an analytical continuation.

We can also obtain the perturbative inversion of (11) by substituting $a_{\text {pert }}=$ $\sum_{m=0}^{\infty} \beta^{m} a_{m}(t)$ as well as its time derivative. Since the Einstein solution, $a_{o}(t)$, is different from zero, we can substitute

$$
\left(\frac{1+\dot{a}^{2} / N^{2}}{a^{2}}\right)_{\text {pert }}=\sum_{m=0}^{\infty} \beta^{m} f_{m}
$$

instead. We conclude, after some manipulations, that $f_{M} \propto\left(T_{11} / L_{1} N^{2}\right)^{m+1}$, and thus

$$
\left[\left(\frac{1+\dot{a}^{2} / N^{2}}{a^{2}}\right)_{\text {pert }}\right]_{\mid \frac{T_{11}}{L_{1} N^{2}}=0}=0
$$

which is precisely condition (14) on the inversion $P_{1}^{-1}$. Following the same line of reasoning as before, it is easily seen that $P_{1}^{-1}$ then provides the generalized perturbative inversion of the Hamiltonian constraint (11). 


\section{Instanton solutions}

We will now apply the procedure given in section 2 to some matter fields models which give rise to instanton solutions, that is classical Euclidean solutions that can be interpreted as a tunnelling between two Lorentzian regions. We will consider the case of an axionic field and that of a conformal scalar field.

For a spherically symmetric axionic field, Myers has shown [11] that the corresponding Lorentzian action on the classical solution for such a field can be written

$$
S_{\text {mat }}=-L_{1} A_{D-1} \int \mathrm{d} t N \frac{\hat{m}^{2}}{a^{D-1}}
$$

$\dot{m}$ being a certain real constant.

In the case of a homogeneous scalar field conformally coupled to the HilbertEinstein term, the action of the matter field can be shown to take the form

$$
S_{\text {mat }}=L_{1} A_{D-1} \int \mathrm{d} t \frac{N}{a}\left[a^{2} \frac{\dot{\chi}^{2}}{N^{2}}-\frac{(D-2)^{2}}{4} \chi^{2}\right] .
$$

Here, $\chi=\phi a^{(D-2) / 2}$, and the conformal scalar field $\phi$ has been conveniently normalized. Therefore (21) generalizes to $D$-dimensions the usual expression for fourdimensional spacetimes [23].

We will also allow the presence of a positive cosmological constant, included in the matter part of the system [11, 12],

$$
S_{\Lambda}=-L_{1} A_{D-1} \int \mathrm{d} t N a^{D-1} \hat{\Lambda}
$$

where, following Myers [11], we have redefined the cosmological constant, $\hat{\Lambda}=$ $2 \Lambda /(D-1)(D-2)$.

By means of the Wick rotation [24], $N \rightarrow-\mathrm{i} N$, we obtain the following Euclidean Hamil tonian constraints:

(i) Axionic field plus cosmological constant:

$$
P\left(\frac{1-\dot{a}^{2} / N^{2}}{a^{2}}\right)=\frac{\hat{m}^{2}}{a^{2 D-2}}+\hat{\Lambda}
$$

(ii) Conformal scalar field plus cosmological constant:

$$
P\left(\frac{1-\dot{a}^{2} / N^{2}}{a^{2}}\right)=\frac{1}{a^{D}}\left(\frac{(D-2)^{2}}{4} \chi^{2}-a^{2} \frac{\dot{\chi}^{2}}{N^{2}}\right)+\hat{\Lambda} .
$$

In the latter case, the field $\chi$ must also verify the dynamical equation

$$
\frac{(D-2)^{2}}{4} \chi-\frac{a}{N} \frac{\mathrm{d}}{\mathrm{d} t}\left(a \frac{\dot{\chi}}{N}\right)=0
$$

whose general solution, as a function of the conformal time $\eta(a \mathrm{~d} \eta=N \mathrm{~d} t)$, turns out to be

$$
\chi(\eta)=A \sinh \left(\frac{D-2}{2} \eta\right)+B \cosh \left(\frac{D-2}{2} \eta\right) .
$$


Denoting $k=\left(B^{2}-A^{2}\right)(D-2)^{2} / 4$ we finally get

$$
P\left(\frac{1-\dot{a}^{2} / N^{2}}{a^{2}}\right)=\frac{k}{a^{D}}+\hat{\Lambda} .
$$

In the gauge $N=1,(23)$ and (27) can be expressed in the form

$$
P\left(\frac{1-\dot{a}^{2}}{a^{2}}\right)=\frac{k}{a^{2 \alpha}}+\hat{\Lambda} \quad \text { with } \alpha>M
$$

where $k$ is as previously defined for the conformal case, and is equal to $\hat{m}^{2}$ for the axionic case. Note that the parameter $\alpha$ is $D / 2$ for the conformal case and $(D-1)$ for the axionic case, so that in both cases $\alpha>M$.

In order to obtain the classical solution to (28) in the physical region $a>0$ by applying the inversion $P_{1}^{-1}$, one must require $k \geq 0$. Although the restriction to positive $k$ reduces the applicability of our procedure, we do not consider this limitation as a serious shortcoming of our model. In fact, even Einstein gravity presents important problems in the corresponding models for negative $k$ [23]; for instance, the Euclidean solutions to the Einstein Hamiltonian constraint, $\left(1-\dot{a}^{2}\right) / a^{2}=k a^{-2 \alpha}+\hat{\Lambda}$, reach the origin, $a=0$, in a singular way if $k<0$.

We will restrict ourselves in what follows to the cases of positive $k$. According to our interpretation of Lovelock theories, the classical solution to the constraint (28) is given by

$$
\frac{1-\dot{a}^{2}}{a^{2}}=P_{1}^{-1}\left(\frac{k}{a^{2 \alpha}}+\hat{\Lambda}\right)>0 .
$$

The last inequality comes from $k a^{-2 \alpha}+\hat{\Lambda}>0$ and $P_{1}^{-1}(z)>0$ if $z>0$.

In the regions in which a Euclidean solution exists, $\dot{a}^{2} \geq 0$, and thus

$$
\frac{1}{a^{2}} \geq P_{1}^{-1}\left(\frac{k}{a^{2 \alpha}}+\hat{\Lambda}\right) .
$$

Since $P(x)$ is a strictly increasing function in all $x \geq 0$ it follows, then, that

$$
P\left(\frac{1}{a^{2}}\right) \geq \frac{k}{a^{2 \alpha}}+\hat{\Lambda}
$$

in such regions.

We will study first the case of the vanishing cosmological constant. Denoting $y=$ $a^{-2}>0$, we want to determine the intervals of the positive real axis in which $P(y) \geq$ $k y^{\alpha}$. As $P(y)$ is a polynomial of degree $M<\alpha, P(y)<k y^{\alpha}$ for sufficiently large $y$, whereas $P(y)>k y^{\alpha}$ for sufficiently small $y$. Then, these two functions intersect one another at least once in the positive real axis, and the number of intersection points in this interval is, in general, odd. These intersection points are the solutions of

$$
P(y)-k y^{\alpha}=0 \quad \text { for } y>0
$$

According to our discussion, equation (32) admits an odd number of solutions, except for multiple roots. We can now order and index these intersection points: $y_{1} \leq$ 
$y_{2} \leq \ldots \leq y_{2 n+1}$ (equal sign only when (32) has a multiple root). Then, in terms of the scale factor $a$, the regions of Euclidean regime, in which $P\left(a^{-2}\right) \geq k a^{-2 \alpha}$, are given by the intervals $\left(1 / \sqrt{y_{2 n+1}}, 1 / \sqrt{y_{2 n}}\right), \ldots,\left(1 / \sqrt{y_{1}}, \infty\right)$. At the points $a_{i}=1 / \sqrt{y_{i}}$, $i=1, \ldots, 2 n+1, \dot{a}$ vanishes, and so does the extrinsic curvature of the corresponding surfaces of constant time. Hence, these surfaces correspond to extremal surfaces. On them, one can analytically continue back to the Lorentzian signature, thus returning to the Lorentzian regime. The instanton solutions connect the resulting Lorentzian regions to one another and, therefore, can be interpreted as representing tunnelling solutions. In order to study the behaviour of the axionic and conformal scalar fields in the tunnelling process one should follow the same lines as for the corresponding Hilbert-Einstein cases [11, 22], In a similar way, given a certain instanton which covers one of the mentioned intervals $\left(1 / \sqrt{y_{2 i+1}}, 1 / \sqrt{y_{2 i}}\right), i=0, \ldots, n$, one can sew together a number of these Euclidean manifolds by joining them on their extremal surfaces of the same value of the scale factor [12].

Of greater interest is the solution which results from sewing together two instantons which cover the region $\left(1 / \sqrt{y_{1}}, \infty\right)^{-}$by their minimal surfaces. The geometry of this solution presents two asymptotically flat regions connected by a throat of minimum radius, $a_{\min }=1 / \sqrt{y_{1}}$.

In the axionic field case, a compact expression for the Euclidean action of this wormhole can be easily obtained. We first carry out the Wick rotation in Lorentzian action, defining the Euclidean action by $I(N)=-i S(-i N)$. Since our solution is asymptotically flat, in order to render the total surface contribution on its boundaries finite, we have to add new surface terms to the action (6), as mentioned in section 2 . In the asymptotic limit, for our solution, these new terms cancel those that had been already considered. From (5) and (7), and using the Hamiltonian constraint

$$
P\left(\frac{1-\dot{a}^{2}}{a^{2}}\right)=\frac{\hat{m}^{2}}{a^{2 D-2}}
$$

we get

$$
I=4 L_{1} A_{D-1} \int_{a_{\min }}^{\infty} \mathrm{d} a a^{D-2} \frac{\ddot{a}}{\dot{a}} \sum_{m=1}^{M} \frac{\hat{L}_{m} m}{D-2 m}\left(\frac{1-\dot{a}^{2}}{a^{2}}\right)^{m-1}
$$

where $\ddot{a}$ and $\dot{a}$ must be expressed in terms of the scale factor $a$ for the solution.

After differentiating (33), we obtain $\ddot{a}$ as a function of $a$ and $\dot{a}$. Then, by substituting $\dot{a}=\sqrt{1-a^{2} P_{1}^{-1}\left(\hat{m}^{2} a^{2-2 D}\right)}$ and by making the following change of variable: $z=P_{1}^{-1}\left(\hat{m}^{2} a^{2-2 D}\right)$, we get finally from (34)

$$
\begin{aligned}
I=\frac{2 L_{1} A_{D-1}}{D-1} & \int_{0}^{1 / a_{\mathrm{min}}^{2}} \mathrm{~d} z\left(\frac{\hat{m}^{2}}{P(z)}\right)^{D / 2(D-1)} \frac{1}{\sqrt{1-z\left(\hat{m}^{2} / P(z)\right)^{1 / D-1}}} \\
& \times\left[D-1-\frac{z Q(z)}{P(z)}\right] \int_{0}^{1} \mathrm{~d} x x^{D-3} Q\left(\frac{z}{x^{2}}\right)
\end{aligned}
$$

where we have employed the equality

$$
\sum_{m=1}^{M} \frac{\hat{L}_{m}^{m}}{D-2 m} y^{m-1}=\int_{0}^{1} \mathrm{~d} x x^{D-3} Q\left(\frac{y}{x^{2}}\right) .
$$


To perform the explicit calculation of the integrals in (35) we need to know the functional form of the polynomial $P(x)$.

Notice that, whereas in Einstein gravity there only exists one extremal surface for the kind of models we are considering, which are given by

$$
\frac{1}{a_{\min }^{2}}=\frac{k}{a_{\min }^{2 \alpha}} \Rightarrow a_{\min }=k^{1 / 2(\alpha-1)}
$$

in Lovelock gravity the number of extremal surfaces could be larger than one. There is no previous guarantee of (32) having a unique solution. This result is analogous to that which was found by Whitt [17] in studying the horizon problem in Lovelock gravity for his perturbed Schwarzschild solution. The horizons of that solution are localized on the surfaces of $r=r_{h}$, where

$$
P\left(\frac{1}{r_{h}^{2}}\right)=\frac{\mu}{L_{1}} \frac{1}{r_{h}^{D-1}} \quad \text { and } r_{h}>0
$$

with $\mu A_{D-2}>0$ being the black hole mass. Denoting $y=r_{h}^{-2}$ and $k=\mu / L_{1}$, (38) can be rewritten as $P(y)=k y^{(D-1) / 2}$. This equation has the general form (32), with $\alpha=(D-1) / 2$ larger than the degree of the polynomial $P(y)$, except for the case of odd $D$ and $\hat{L}_{(D-1) / 2} \neq 0$. For in that case, the solution presented a naked singularity for sufficiently small black hole masses. In all other cases, there was always at least one horizon for any black hole mass, although, in contrast to the Einstein case, one cannot guarantee the uniqueness of this horizon.

We can assure the uniqueness of the solution of (32) for at least two cases:

(i) when all the coefficients $\hat{L}_{m}$, with $m>1$, are sufficiently small, that is for pure perturbative theory; and

(ii) when all the $\hat{L}_{m}$ are positive.

In the first case, the terms $\sum_{m=2}^{M} \hat{L}_{m} y^{m}$ which appear in (32) can be treated as regular perturbations [25] of the equation

$$
y-k y^{\alpha}=0
$$

because $\alpha>M$. If all the coefficients $\hat{L}_{m}(m>1)$ are small enough, the effect of these perturbations is only to slightly modify the solutions to (39) and since they respect the solution $y=0$, there cannot appear any new positive real solution, the unique one being that corresponding to the perturbation of $k^{-1 /(\alpha-1)}$. Similarly, we conclude that in this case $P(y)-k y^{\alpha}$ has a unique extremum in $y>0$, because the possible extrema are given by the solutions of the equation

$$
\sum_{m=2}^{M} \hat{L}_{m} m y^{m-1}+1-k \alpha y^{\alpha-1}=0
$$

in which the Lovelock corrections are regular perturbations.

The solution of (32) is also unique whenever all the $\hat{L}_{m}$ are positive. In this case, the condition of $P(x)$ being a strictly increasing polynomial for all $x \geq 0$ turns out to be redundant. We know that for sufficiently small $y, P(y)>k y^{\alpha}$, but also that these two functions intersect one another at least once in $y>0$. In order to make sure of the uniqueness of the intersection point it suffices to show that, for all the points $y>0$ 
where $k y^{\alpha} \geq P(y)$, the derivative $k \alpha y^{\alpha-1}$ is larger than that of $P(y), Q(y)$, because then, once the intersection point has been reached, $k y^{\alpha}$ will increase faster than $P(y)$, and they will not gather anymore. But, if $k y^{\alpha} \geq P(y)$, we have

$$
k \alpha y^{\alpha-1} \geq \alpha \frac{P(y)}{y}=\alpha \sum_{m=1}^{M} \hat{L}_{m} y^{m-1}>\sum_{m=1}^{M} \hat{L}_{m} m y^{m-1}=Q(y)
$$

the last inequality arising from $\alpha>M \geq m$ and $\hat{L}_{m}$ and $y$ being positive. We can also show that, in this case, $P(y)-k y^{\alpha}$ has a unique extremum in positive $y$, given by

$$
Q(y)-k \alpha y^{\alpha-1}=0 \quad \text { with } y>0
$$

For sufficiently small $y, Q(y)>k \alpha y^{\alpha-1}$, but $k \alpha y^{\alpha-1}>Q(y)$ for large $y$, so that there is at least a solution of (42). This solution is unique because, if $k \alpha y^{\alpha-1} \geq Q(y)$,

$k \alpha(\alpha-1) y^{\alpha-2} \geq(\alpha-1) \sum_{m=1}^{M} \hat{L}_{m} m y^{m-2}>\sum_{m=2}^{M} \hat{L}_{m} m(m-1) y^{m-2}=Q^{\prime}(y)$

which allows us to apply similar arguments to before.

Thus, at least for the two considered cases, there exists a unique solution $y_{1}$ of equation (32). It follows that, in at least these cases, models whose gravitational dynamics is governed by equation (28) with $\hat{\Lambda}=0$, admit only one instanton solution, covering the region $\left(1 / \sqrt{y_{1}}, \infty\right)$, from which one can obtain a wormhole. In a similar way, by the discussion following (38), one can also assure the existence and uniqueness of the horizon for the perturbed Schwarzschild solution dealt with by Whitt for the above two cases, except when $D$ is odd and $\hat{L}_{(D-1) / 2} \neq 0$. It is in this sense that we can consider the physics in all these situations as being equivalent to that of Einstein gravity.

Consider now models with a non-vanishing cosmological constant. In general, $P(y)-k y^{\alpha}$ will have at least an extremum in each interval $\left(0, y_{1}\right), \ldots,\left(y_{2 n}, y_{2 n+1}\right)$ (except when (32) admits a multiple root: $y_{2 i}=y_{2 i+1}, i \in\{1, \ldots, n\}$ ). Let us denote $\bar{y}_{1}, \ldots, \bar{y}_{j}$ and $\breve{y}_{1}, \ldots, \breve{y}_{1}$ the maxima and minima of $P(y)-k y^{\alpha}$, respectively, in the union of these intervals (in general, $j=l+n+1$ ). Setting $P\left(\bar{y}_{i}\right)-k \bar{y}_{i}^{\alpha}=\bar{\Lambda}_{i}, i=1, \ldots, j$, and $P\left(\breve{y}_{i}\right)-k \breve{y}_{i}^{\alpha}=\breve{\Lambda}_{i}, i=1, \ldots, l$, let us rearrange $\bar{\Lambda}_{i}$ and $\breve{\Lambda}_{i}$ (and, thereby, $\bar{y}_{i}$ and $\breve{y}_{i}$ ) in such a manner that $\bar{\Lambda}_{1} \geq \bar{\Lambda}_{2} \geq \ldots \geq \bar{\Lambda}_{j}$ and $\breve{\Lambda}_{1} \geq \breve{\Lambda}_{2} \geq \ldots \geq \breve{\Lambda}_{1}$. Then, if $\hat{\Lambda} \geq \bar{\Lambda}_{1}$, we have $P(y)-k y^{\alpha} \leq \hat{\Lambda}$ for all positive $y$. Thus, there is neither Euclidean regime nor instanton solutions in this case. Instanton solutions are present only for $\bar{\Lambda}_{1}>\hat{\Lambda} \geq 0$, and, if $\bar{\Lambda}_{p}>\hat{\Lambda} \geq \bar{\Lambda}_{p+1}, 1 \geq p \geq j$, and $\breve{\Lambda}_{q}>\hat{\Lambda} \geq \breve{\Lambda}_{q+1}, 0 \geq q \geq l$ (with $\breve{\Lambda}_{0}=\bar{\Lambda}_{1}$ and $\bar{\Lambda}_{j+1}=0=\breve{\Lambda}_{l+1}$ ), the number of different regions in which an instanton solution exists is equal to $p-q$.

When $P(y)-k y^{\alpha}$ has only a zero and a unique maximum in the positive real axis, that is when all the coefficients $\hat{L}_{m}$ are positive or sufficiently small for $m>$ 1 , for instance, the situation turns out to be much simpler, and equivalent to that corresponding to Einstein gravity. Denoting the maximum by $\bar{y}_{1}$ and $P\left(\bar{y}_{1}\right)-k \bar{y}_{1}^{\alpha}=$ $\bar{\Lambda}_{1}$, it follows that there is only one instanton solution for $\bar{\Lambda}_{1}>\hat{\Lambda} \geq 0$. This Euclidean manifold covers the region $a \in\left(1 / \sqrt{y_{a}}, 1 / \sqrt{y_{b}}\right)$, with $y_{a}, y_{b}\left(y_{a}>y_{b}\right)$ being the two 
solutions of $P(y)=k y^{\alpha}+\hat{\Lambda}$ in $y>0$. It is readily seen that the two surfaces of constant time with scale factors $1 / \sqrt{y_{a}}$ and $1 / \sqrt{y_{b}}$ are then extremal surfaces. This instanton can be interpreted as a tunnelling between a baby universe in the minimal surface $a=1 / \sqrt{y_{a}}$ and an asymptotically de Sitter universe $(\hat{\Lambda}>0)$ in the maximal surface $a=1 / \sqrt{y_{b}}$. Sewing together several of these instantons by their extremal surfaces, we can also form other Euclidean manifolds, corresponding to different types of tunnelling.

In conclusion, by treating Lovelock gravity as a perturbative theory, we have carried out a general study of a homogeneous and isotropic minisuperspace model with a. matter content which produces instanton type solutions. We have particularized this study to the not very restrictive case in which the Lovelock polynomial (7) is strictly increasing in the positive real axis. In some more specific cases (namely, when all the Lovelock coefficients $\hat{L}_{m}$, with $m>1$, are sufficiently small or when all the $\hat{L}_{m}$ are positive) we have shown that, if the cosmological constant is not very large, the instanton solution is essentially unique; for larger cosmological constants there are not instanton solutions. This is in complete parallelism with the corresponding Einstein gravity models.

In order to perform explicit calculations, we need to know the Lovelock coefficients $\hat{L}_{m}$ or, equivalently, the polynomial $P(x)$. Even if this information is provided, most of these calculations should be done numerically.

\section{Acknowledgments}

I would like to thank P F González Díaz for his fruitful comments and collaboration. I am also grateful to $\mathrm{L} J \mathrm{~J}$ Garay and $\mathrm{L} M$ Campbell for their encouraging comments and to $\overline{\mathrm{J}} \mathrm{A}$ Torresano for technical help.

\section{References}

[1] Müller-Hoissen F 1985 Phys. Letl. 163B 106; 1986 Class. Quantum Grav. 3665

[2] Lovelock D 1971 J. Math. Phys. 12498

[3] Zwiebach B 1985 Phys. Lett. 150B 315

[4] Zumino B 1986 Phys. Rep. 137109

[5] Simon J Z 1989 Preprint University of California UCSB-TH-89-50a

[6] Mena Marugán G A 1990 Phys. Rev. D 422607

[7] Henneaux M, Teitelboim C and Zanellj J 1987 Phys. Rev, A 364417

[8] Teitelboim $\mathrm{C}$ and Zanelli J 1987 Class. Quantum Grav. 4125

[9] Choquet-Bruhat Y 1988 J. Math. Phys. 291981

[10] Eguchi T, Gilkey P B and Hanson A I 1980 Phys. Rep. 66 213

[11] Myers R C 1989 Nucl. Phys. B 323225

[12] Myers R C 1988 Phys, Rev. D 381327

[13] Wheeler J T 1986 Nucl. Phys. B 268 737; 1986 Nucl. Phys. B 273732

[14] Myers R C and Simon J Z 1988 Phys. Rev. D 382434

[15] Deruelle N and Farina-Busto L 1990 Phys. Rev. D 413696

[16] Boulware D G and Deser S 1985 Phys. Rev. Lett. 552656

[17] Whitt B 1988 Phys. Rev. D 383000

[18] Myers R C 1987 Phys. Rev. D 36392

[19] Gibbons G W and Hawking S W 1977 Phys. Reu. D 152752

[20] Hawking S W 1979 General Relativily: An Einstein Centenary Survey ed S W Hawking and W Israel (Cambridge: Carnbridge University Press)

[21] Ahlfors L V 1979 Complex Analysis (New York: McGraw-Hill) 
[22] Jaén X, Llosa J and Molina A 1986 Phys. Rev. D 342302

[23] Halliwell J J and Laflamme R 1989 Class. Quantum Grov, 61839

[24] Hartle J B and Hawking S W 1983 Phys, Rev. D 282960

[25] Bender C M and Orszag S A 1978 Advanced Mathematical Methods for Scientists and Engineers (Singapore: McGraw-Hill) 